\title{
RATE OF CONVERGENCE OF BETA OPERATORS \\ OF SECOND KIND FOR FUNCTIONS WITH \\ DERIVATIVES OF BOUNDED VARIATION
}

VIJAY GUPTA, ULRICH ABEL, AND MIRCEA IVAN

Received 23 May 2005 and in revised form 12 September 2005

We study the approximation properties of beta operators of second kind. We obtain the rate of convergence of these operators for absolutely continuous functions having a derivative equivalent to a function of bounded variation.

\section{Introduction}

For Lebesgue integrable functions $f$ on the interval $I=(0, \infty)$, beta operators $L_{n}$ of second kind are given by

$$
\left(L_{n} f\right)(x)=\frac{1}{B(n x, n+1)} \int_{0}^{\infty} \frac{t^{n x-1}}{(1+t)^{n x+n+1}} f(t) d t .
$$

Obviously the operators $L_{n}$ are positive linear operators on the space of locally integrable functions on $I$ of polynomial growth as $t \rightarrow \infty$, provided that $n$ is sufficiently large.

In 1995, Stancu [10] gave a derivation of these operators and investigated their approximation properties. We mention that similar operators arise in the work by Adell et al. $[3,4]$ by taking the probability density of the inverse beta distribution with parameters $n x$ and $n$.

Recently, Abel [1] derived the complete asymptotic expansion for the sequence of operators (1.1). In [2], Abel and Gupta studied the rate of convergence for functions of bounded variation.

In the present paper, the study of operators (1.1) will be continued. We estimate their rate of convergence by the decomposition technique for absolutely continuous functions $f$ of polynomial growth as $t \rightarrow+\infty$, having a derivative $f^{\prime}$ coinciding a.e. with a function which is of bounded variation on each finite subinterval of $I$.

Several researchers have studied the rate of approximation for functions with derivatives of bounded variation. We mention the work of Bojanić and Chêng (see $[5,6])$ who estimated the rate of convergence with derivatives of bounded variation for Bernstein and Hermite-Fejer polynomials by using different methods. Further papers on the subject were written by Bojanić and Khan [7] and by Pych-Taberska [9]. See also the very recent paper by Gupta et al. [8] on general class of summation-integral type operators. 


\section{Stancu beta operators}

For the sake of convenient notation in the proofs we rewrite operators (1.1) as

$$
\left(L_{n} f\right)(x)=\int_{0}^{\infty} K_{n}(x, t) f(t) d t
$$

where the kernel function $K_{n}$ is given by

$$
K_{n}(x, t)=\frac{1}{B(n x, n+1)} \frac{t^{n x-1}}{(1+t)^{n x+n+1}} .
$$

Moreover, we put

$$
\lambda_{n}(x, y)=\int_{0}^{y} K_{n}(x, t) d t \quad(y \geq 0) .
$$

Note that $0 \leq \lambda_{n}(x, y) \leq 1(y \geq 0)$.

Our main result is contained in Section 3, while the next section contains some auxiliary results.

\section{Auxiliary results}

For fixed $x \in I$, define the function $\psi_{x}$, by $\psi_{x}(t)=t-x$. The first central moments for the operators $L_{n}$ are given by

$$
\left(L_{n} \psi_{x}^{0}\right)(x)=1, \quad\left(L_{n} \psi_{x}^{1}\right)(x)=0, \quad\left(L_{n} \psi_{x}^{2}\right)(x)=\frac{x(1+x)}{n-1}
$$

(see [1, Proposition 2]). In general, we have the following result.

Lemma 2.1 [1, Proposition 2]. Let $x \in I$ be fixed. For $r=0,1,2, \ldots$ and $n \in \mathbb{N}$, the central moments for the operators $L_{n}$ satisfy

$$
\left(L_{n} \psi_{x}^{r}\right)(x)=O\left(n^{-\lfloor(r+1) / 2\rfloor}\right) \quad(n \longrightarrow \infty)
$$

In view of (1.2), an application of the Schwarz inequality, for $r=0,1,2, \ldots$, yields

$$
\left(L_{n}\left|\psi_{x}^{r}\right|\right)(x) \leq \sqrt{\left(L_{n} \psi_{x}^{2 r}\right)(x)}=O\left(n^{-r / 2}\right) \quad(n \longrightarrow \infty) .
$$

In particular, by (2.1) we have

$$
\left(L_{n}\left|\psi_{x}\right|\right)(x) \leq \sqrt{\frac{x(1+x)}{(n-1)}} .
$$


Lemma 2.2 [2, Proposition 2]. Let $x \in I$ be fixed and $K_{n}(x, t)$ be defined by (1.3). Then, for $n \geq 2$,

$$
\begin{aligned}
\lambda_{n}(x, y) & =\int_{0}^{y} K_{n}(x, t) d t \leq \frac{x(1+x)}{(n-1)(x-y)^{2}} \quad(0 \leq y<x), \\
1-\lambda_{n}(x, z) & =\int_{z}^{\infty} K_{n}(x, t) d t \leq \frac{x(1+x)}{(n-1)(z-x)^{2}} \quad(x<z<\infty) .
\end{aligned}
$$

\section{The main result}

Throughout this paper, for each function $g$ of bounded variation on $I$ and fixed $x \in I$, we define the auxiliary function $g_{x}$, which is given by

$$
g_{x}(t)= \begin{cases}g(t)-g(x-) & (0 \leq t<x), \\ 0 & (t=x), \\ g(t)-g(x+) & (x<t<\infty) .\end{cases}
$$

Furthermore, $\bigvee_{a}^{b}(g)$ denotes the total variation of $g$ on $[a, b]$. For $r \geq 0$, let $D B_{r}(I)$ be the class of all absolutely continuous functions $f$ defined on $I$,

(i) having on $I$ a derivative $f^{\prime}$ coinciding a.e. with a function which is of bounded variation on each finite subinterval of $I$,

(ii) satisfying $f(t)=O\left(t^{r}\right)$ as $t \rightarrow+\infty$.

Note that all functions $f \in D B_{r}(I)$ possess, for each $a>0$, a representation

$$
f(x)=f(a)+\int_{a}^{x} \psi(t) d t \quad(x \geq a)
$$

with a function $\psi$ of bounded variation on each finite subinterval of $I$.

The following theorem is our main result.

Theorem 3.1. Let $r \in \mathbb{N}, x \in I$, and $f \in D B_{r}(I)$. Then there holds

$$
\begin{aligned}
\left|\left(L_{n} f\right)(x)-f(x)\right| \leq & \frac{1}{2} \sqrt{\frac{x(1+x)}{n-1}}\left|f^{\prime}(x+)-f^{\prime}(x-)\right|+\frac{x}{\sqrt{n}} V_{x-x / \sqrt{n}}^{x+x / \sqrt{n}}\left(\left(f^{\prime}\right)_{x}\right) \\
& +\frac{1+x}{n-1}\left(\sum_{k=1}^{\lfloor\sqrt{n}\rfloor} V_{x-x / k}^{x+x / k}\left(\left(f^{\prime}\right)_{x}\right)+x^{-1}|f(2 x)-f(x)|+2\left|f^{\prime}(x+)\right|\right) \\
& +\frac{c_{r, x} \cdot M_{r, x}(f)}{n^{r / 2}},
\end{aligned}
$$

where the constants $c_{r, x}$ and $M_{r, x}(f)$ are given by

$$
\begin{aligned}
c_{r, x} & =\sup _{n \in \mathbb{N}} \sqrt{n^{r}\left(L_{n} \psi_{x}^{2 r}\right)(x)}, \\
M_{r, x}(f) & =2^{r} \sup _{t \geq 2 x} t^{-r}|f(t)-f(x)| .
\end{aligned}
$$


Remark 3.2. Note that, for each $f \in D B_{r}(I)$, we have $M_{r, x}(f)<+\infty$. Furthermore, Lemma 2.1 implies that $c_{r, x}<+\infty$.

Proof. For $x \in I$, we have

$$
\left(L_{n} f\right)(x)-f(x)=\int_{0}^{\infty} K_{n}(x, t)(f(t)-f(x)) d t=\int_{0}^{\infty} K_{n}(x, t) \int_{x}^{t} f^{\prime}(u) d u d t .
$$

Now we take advantage of the identity

$$
\begin{aligned}
f^{\prime}(u)= & \left(f^{\prime}\right)_{x}(u)+\frac{1}{2}\left(f^{\prime}(x+)+f^{\prime}(x-)\right)+\frac{1}{2}\left(f^{\prime}(x+)-f^{\prime}(x-)\right) \operatorname{sign}(u-x) \\
& +\left(f^{\prime}(x)-\frac{1}{2}\left(f^{\prime}(x+)+f^{\prime}(x-)\right)\right) \chi_{x}(u)
\end{aligned}
$$

where $\chi_{x}(u)=1(u=x)$ and $\chi_{x}(u)=0(u \neq x)$. Obviously, we have

$$
\int_{0}^{\infty} K_{n}(x, t) \int_{x}^{t}\left(f^{\prime}(x)-\frac{1}{2}\left(f^{\prime}(x+)+f^{\prime}(x-)\right)\right) \chi_{x}(u) d u d t=0 .
$$

Furthermore, by (2.1) and (2.4), respectively, we have

$$
\begin{aligned}
\int_{0}^{\infty} K_{n}(x, t) \int_{x}^{t} \frac{1}{2}\left(f^{\prime}(x+)\right. & \left.+f^{\prime}(x-)\right) d u d t=\frac{1}{2}\left(f^{\prime}(x+)+f^{\prime}(x-)\right) \int_{0}^{\infty} K_{n}(x, t)(t-x) d t=0 \\
\left|\int_{0}^{\infty} K_{n}(x, t) \int_{x}^{t} \frac{1}{2}\left(f^{\prime}(x+)-f^{\prime}(x-)\right) \operatorname{sign}(u-x) d u d t\right| & \leq \frac{1}{2}\left|f^{\prime}(x+)-f^{\prime}(x-)\right| \int_{0}^{\infty} K_{n}(x, t)|t-x| d t \\
& \leq \frac{1}{2} \sqrt{\frac{x(1+x)}{n-1}}\left|f^{\prime}(x+)-f^{\prime}(x-)\right|
\end{aligned}
$$

Collecting the latter relations, we obtain the estimate

$$
\left|\left(L_{n} f\right)(x)-f(x)\right| \leq\left|A_{n}(f, x)+B_{n}(f, x)+C_{n}(f, x)\right|+\frac{1}{2} \sqrt{\frac{x(1+x)}{n-1}}\left|f^{\prime}(x+)-f^{\prime}(x-)\right|
$$

with the denotations

$$
\begin{aligned}
& A_{n}(f, x)=\int_{0}^{x} K_{n}(x, t) \int_{x}^{t}\left(f^{\prime}\right)_{x}(u) d u d t, \\
& B_{n}(f, x)=\int_{x}^{2 x} K_{n}(x, t) \int_{x}^{t}\left(f^{\prime}\right)_{x}(u) d u d t, \\
& C_{n}(f, x)=\int_{2 x}^{\infty} K_{n}(x, t) \int_{x}^{t}\left(f^{\prime}\right)_{x}(u) d u d t .
\end{aligned}
$$

In order to complete the proof, it is sufficient to estimate the terms $A_{n}(f, x), B_{n}(f, x)$, and $C_{n}(f, x)$. 
Using integration by parts, and application of Lemma 2.2 yields

$$
\begin{aligned}
\left|A_{n}(f, x)\right| & =\left|\int_{0}^{x} \int_{x}^{t}\left(f^{\prime}\right)_{x}(u) d u d_{t} \lambda_{n}(x, t)\right|=\left|\int_{0}^{x} \lambda_{n}(x, t)\left(f^{\prime}\right)_{x}(t) d t\right| \\
& \leq\left(\int_{0}^{x-x / \sqrt{n}}+\int_{x-x / \sqrt{n}}^{x}\right)\left|\lambda_{n}(x, t)\right| V_{t}^{x}\left(\left(f^{\prime}\right)_{x}\right) d t \\
& \leq \frac{x(1+x)}{n-1} \int_{0}^{x-x / \sqrt{n}}(x-t)^{-2} V_{t}^{x}\left(\left(f^{\prime}\right)_{x}\right) d t+\frac{x}{\sqrt{n}} V_{x-x / \sqrt{n}}^{x}\left(\left(f^{\prime}\right)_{x}\right) .
\end{aligned}
$$

By the substitution of $u=x /(x-t)$, we obtain

$$
\begin{aligned}
\int_{0}^{x-x / \sqrt{n}}(x-t)^{-2} V_{t}^{x}\left(\left(f^{\prime}\right)_{x}\right) d t & =x^{-1} \int_{1}^{\sqrt{n}} V_{x-x / u}^{x}\left(\left(f^{\prime}\right)_{x}\right) d u \\
& \leq x^{-1} \sum_{k=1}^{\lfloor\sqrt{n}\rfloor} \int_{k}^{k+1} V_{x-x / u}^{x}\left(\left(f^{\prime}\right)_{x}\right) d u \\
& \leq x^{-1} \sum_{k=1}^{\lfloor\sqrt{n}\rfloor} V_{x-x / k}^{x}\left(\left(f^{\prime}\right)_{x}\right) .
\end{aligned}
$$

Thus we have

$$
\left|A_{n}(f, x)\right| \leq \frac{1+x}{n-1} \sum_{k=1}^{\lfloor\sqrt{n}\rfloor} V_{x-x / k}^{x}\left(\left(f^{\prime}\right)_{x}\right)+\frac{x}{\sqrt{n}} V_{x-x / \sqrt{n}}^{x}\left(\left(f^{\prime}\right)_{x}\right) .
$$

Furthermore, we have

$$
\begin{aligned}
\left|B_{n}(f, x)\right|= & \left|-\int_{x}^{2 x} \int_{x}^{t}\left(f^{\prime}\right)_{x}(u) d u d_{t}\left(1-\lambda_{n}(x, t)\right)\right| \\
\leq & \left|\int_{x}^{2 x}\left(f^{\prime}\right)_{x}(u) d u\right|\left|1-\lambda_{n}(x, 2 x)\right|+\int_{x}^{2 x}\left|\left(f^{\prime}\right)_{x}(t)\right|\left|1-\lambda_{n}(x, t)\right| d t \\
\leq & \frac{1+x}{(n-1) x}\left|f(2 x)-f(x)-x f^{\prime}(x+)\right|+\int_{x}^{x+x / \sqrt{n}} V_{x}^{t}\left(\left(f^{\prime}\right)_{x}\right) d t \\
& +\frac{x(1+x)}{n-1} \int_{x+x / \sqrt{n}}^{2 x}(t-x)^{-2} V_{x}^{t}\left(\left(f^{\prime}\right)_{x}\right) d t,
\end{aligned}
$$

where we applied Lemma 2.2. By the substitution of $u=x /(t-x)$, we obtain

$$
\begin{aligned}
\int_{x+x / \sqrt{n}}^{2 x}(t-x)^{-2} V_{x}^{t}\left(\left(f^{\prime}\right)_{x}\right) d t & =x^{-1} \int_{1}^{\sqrt{n}} V_{x}^{x+x / u}\left(\left(f^{\prime}\right)_{x}\right) d u \\
& \leq x^{-1} \sum_{k=1}^{\lfloor\sqrt{n}\rfloor} \int_{k}^{k+1} V_{x}^{x+x / u}\left(\left(f^{\prime}\right)_{x}\right) d u \\
& \leq x^{-1} \sum_{k=1}^{\lfloor\sqrt{n}\rfloor} V_{x}^{x+x / k}\left(\left(f^{\prime}\right)_{x}\right) .
\end{aligned}
$$


Thus we have

$$
\begin{aligned}
\left|B_{n}(f, x)\right| \leq & \frac{1+x}{(n-1) x}\left|f(2 x)-f(x)-x f^{\prime}(x+)\right|+\frac{1+x}{n-1} \sum_{k=1}^{\lfloor\sqrt{n}\rfloor} V_{x}^{x+x / k}\left(\left(f^{\prime}\right)_{x}\right) \\
& +\frac{x}{\sqrt{n}} V_{x}^{x+x / \sqrt{n}}\left(\left(f^{\prime}\right)_{x}\right) .
\end{aligned}
$$

Finally, we have

$$
\begin{aligned}
\left|C_{n}(f, x)\right| & =\left|\int_{2 x}^{\infty} K_{n}(x, t)\left(f(t)-f(x)-(t-x) f^{\prime}(x+)\right) d t\right| \\
& \leq 2^{-r} M_{r, x}(f) \int_{2 x}^{\infty} K_{n}(x, t) t^{r} d t+\left|f^{\prime}(x+)\right| \int_{2 x}^{\infty} K_{n}(x, t)|t-x| d t .
\end{aligned}
$$

Using the obvious inequalities $t \leq 2(t-x)$ and $x \leq t-x$ for $t \geq 2 x$, we obtain

$$
\begin{aligned}
\left|C_{n}(f, x)\right| & \leq M_{r, x}(f) \int_{2 x}^{\infty} K_{n}(x, t)(t-x)^{r} d t+x^{-1}\left|f^{\prime}(x+)\right| \int_{2 x}^{\infty} K_{n}(x, t)(t-x)^{2} d t \\
& \leq M_{r, x}(f) \cdot\left(L_{n}\left|\psi_{x}^{r}\right|\right)(x)+x^{-1}\left|f^{\prime}(x+)\right|\left(L_{n} \psi_{x}^{2}\right)(x) .
\end{aligned}
$$

By (2.3), we conclude that

$$
\left|C_{n}(f, x)\right|=M_{r, x}(f) \cdot c_{r, x} n^{-r / 2}+\frac{1+x}{n-1}\left|f^{\prime}(x+)\right| .
$$

Combining the estimates (3.13)-(3.19) with (3.9), we get the desired result. This completes the proof of the theorem.

\section{Acknowledgments}

The authors are thankful to the four kind referees for their valuable comments which led to a better presentation of the paper. The revised version of the paper was submitted while the first author was visiting the Department of Mathematics and Statistics, Auburn University, USA, in the fall 2005.

\section{References}

[1] U. Abel, Asymptotic approximation with Stancu beta operators, Rev. Anal. Numér. Théor. Approx. 27 (1998), no. 1, 5-13.

[2] U. Abel and V. Gupta, Rate of convergence of Stancu beta operators for functions of bounded variation, Rev. Anal. Numér. Théor. Approx. 33 (2004), no. 1, 3-9.

[3] J. A. Adell and J. de la Cal, On a Bernstein-type operator associated with the inverse PólyaEggenberger distribution, Rend. Circ. Mat. Palermo (2) Suppl. 33 (1993), 143-154.

[4] J. A. Adell, J. de la Cal, and M. San Miguel, Inverse beta and generalized Bleimann-Butzer-Hahn operators, J. Approx. Theory 76 (1994), no. 1, 54-64.

[5] R. Bojanić and F. Chêng, Rate of convergence of Bernstein polynomials for functions with derivatives of bounded variation, J. Math. Anal. Appl. 141 (1989), no. 1, 136-151. 
[6] Rate of convergence of Hermite-Fejér polynomials for functions with derivatives of bounded variation, Acta Math. Hungar. 59 (1992), no. 1-2, 91-102.

[7] R. Bojanić and M. K. Khan, Rate of convergence of some operators of functions with derivatives of bounded variation, Atti Sem. Mat. Fis. Univ. Modena 39 (1991), no. 2, 495-512.

[8] V. Gupta, V. Vasishtha, and M. K. Gupta, Rate of convergence of summation-integral type operators with derivatives of bounded variation, JIPAM. J. Inequal. Pure Appl. Math. 4 (2003), no. 2 , article $34,1-8$.

[9] P. Pych-Taberska, Pointwise approximation of absolutely continuous functions by certain linear operators, Funct. Approx. Comment. Math. 25 (1997), 67-76.

[10] D. D. Stancu, On the beta approximating operators of second kind, Rev. Anal. Numér. Théor. Approx. 24 (1995), no. 1-2, 231-239.

Vijay Gupta: School of Applied Science, Netaji Subhas Institute of Technology, Azad Hind Fauj Marg, Sector-3, Dwarka, New Delhi-110 045, India

E-mail address: vijay@nsit.ac.in

Ulrich Abel: Fachbereich MND, Fachhochschule Giessen-Friedberg, University of Applied Sciences, Wilhelm-Leuschner-Straße 13, 61169 Friedberg, Germany

E-mail address: ulrich.abel@mnd.fh-friedberg.de

Mircea Ivan: Department of Mathematics, Technical University of Cluj-Napoca, 400020 ClujNapoca, Romania

E-mail address: mircea.ivan@math.utcluj.ro 




Advances in

Operations Research

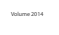

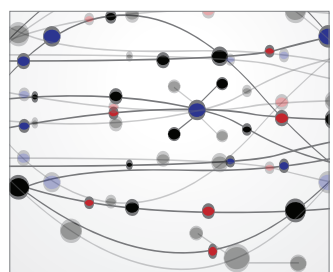

\section{The Scientific} World Journal
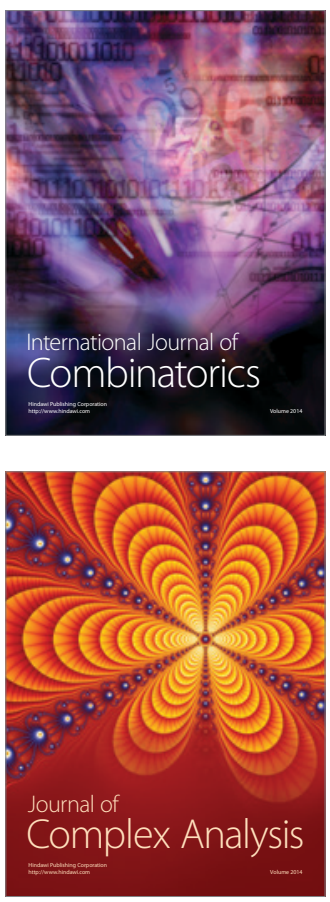

International Journal of

Mathematics and

Mathematical

Sciences
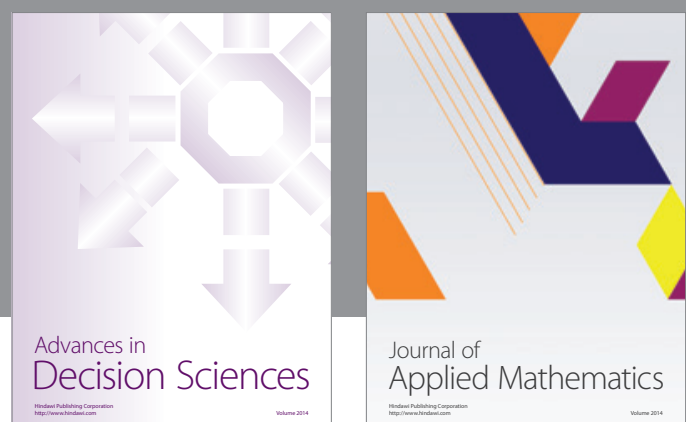

Journal of

Applied Mathematics
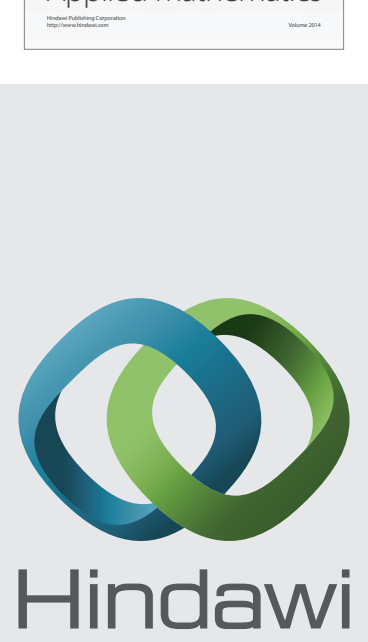

Submit your manuscripts at http://www.hindawi.com
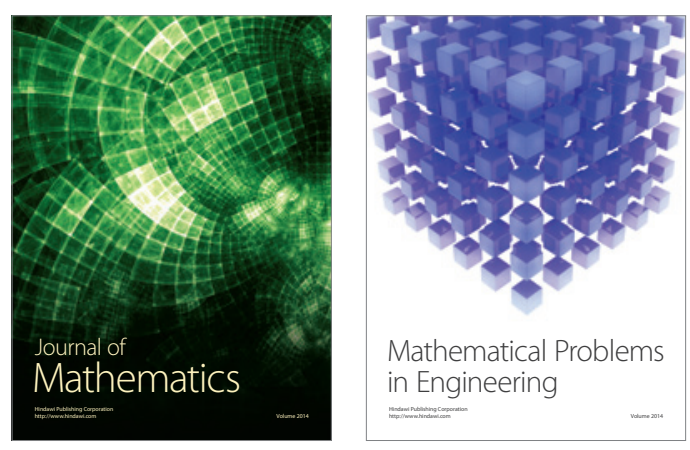

Mathematical Problems in Engineering
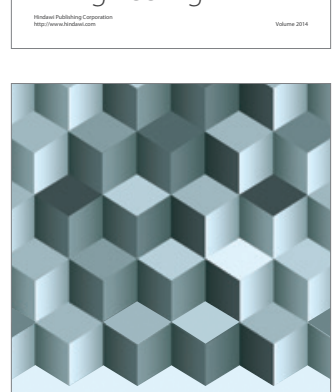

Journal of

Function Spaces
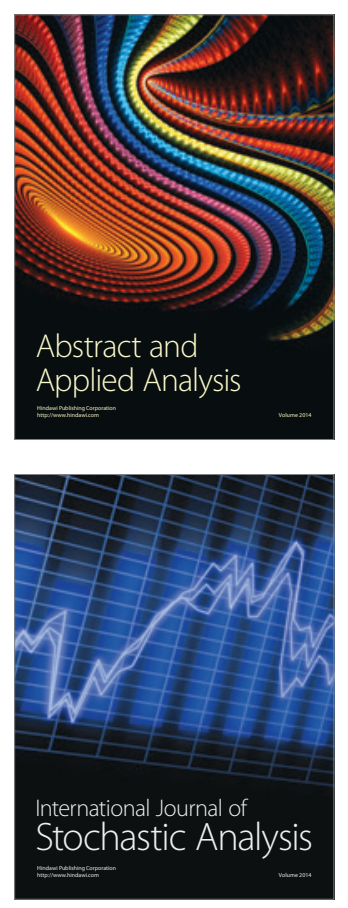

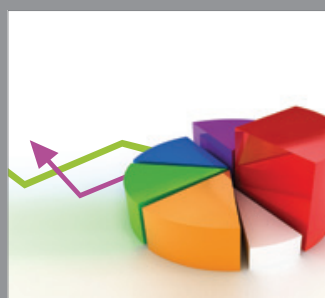

ournal of

Probability and Statistics

Promensencen
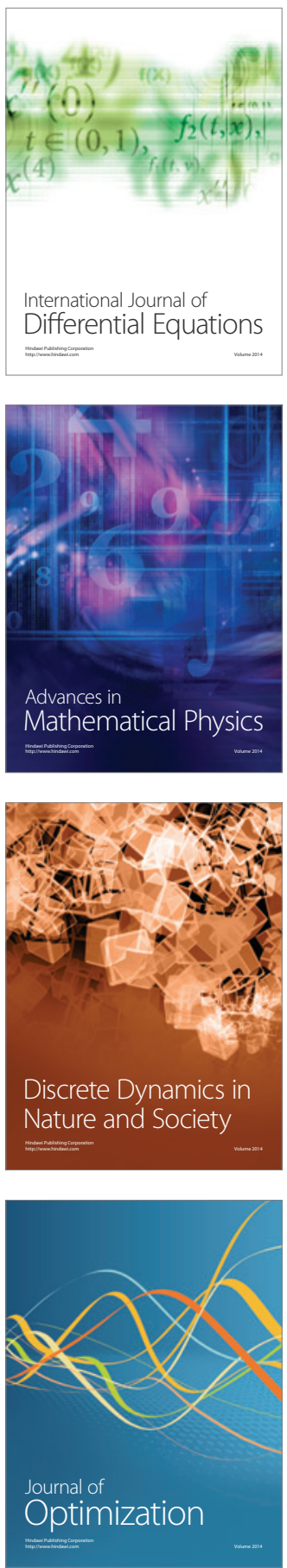